\title{
Estructura y categorías tróficas de peces asociados a praderas de Thalassia testudinum (Hydrocharitales, Hydrocharitaceae) en el golfo de Cariaco, Estado de Sucre, Venezuela*
}

\author{
Thays Allen ${ }^{1}$, Máyré Jiménez ${ }^{1}$ \& Sioliz Villafranca ${ }^{1}$ \\ ${ }^{1}$ Universidad de Oriente, Núcleo de Sucre, Departamento de Biología Marina \\ Instituto Oceanográfico de Venezuela, Avenida Universidad, Cerro Colorado \\ Cumaná, Apartado Postal 245, Código Postal 6101, Estado de Sucre, Venezuela
}

\begin{abstract}
RESUMEN. Se analiza la estructura y categorías tróficas de la comunidad de peces asociados a praderas de Thalassia testudinum del golfo de Cariaco, Venezuela. Las capturas de peces se realizaron desde diciembre 1996 hasta noviembre 1997, utilizando una red tipo chinchorro. Se cuantificaron los individuos, se calculó la abundancia total de las especies, la riqueza específica total y la frecuencia de aparición. Se determinó la categoría trófica de cada especie, estableciéndose cinco categorías: carnívoros, herbívoros, omnívoros, planctívoros y detritívoros. Mediante un dendrograma se estableció el grado de similitud alimentaria entre las distintas especies y su categoría trófica. Se colectaron 15.509 peces pertenecientes a 27 familias y 44 especies. Se determinaron 23 especies carnívoras (57\%), ocho omnívoras (20\%), cinco planctívoras (13\%), dos herbívoras y dos detrítivoras (5\%). De las especies carnívoras se identificaron 12 especies de escasa presencia. Las únicas especies herbívoras fueron Nicholsina usta y Sparisoma radiams. El dendrograma mostró dos grupos principales, el grupo A formado por especies omnívoras y carnívoras y el grupo B, conformado por especies detritívoras, herbívoras y planctívoras.
\end{abstract}

Palabras clave: peces, Thalassia testudinum, categoría trófica, Caribe, Venezuela.

\section{Trophic structure and categories of fish associated with Thalassia testudinum meadows (Hydrocharitales, Hydrocharitaceae) in Golfo de Cariaco, Estado de Sucre, Venezuela*}

\begin{abstract}
The trophic structure and categories of the fish community associated with Thalassia testudinum meadows in the Golfo de Cariaco, Venezuela, were analyzed. The fish were caught from December 1996 through November 1997, using a seine-type net. Specimens were quantified and the total species abundance, total species richness, and frequency of appearance were calculated. Each species was placed into one of five trophic categories: carnivorous, herbivorous, omnivorous, planktivorous, and detritivorous. A dendrogram was used to establish the degree of similarity in food between the different species and trophic categories. The 15,509 fish collected belonged to 27 families and 44 species. Of these, 23 species were found to be carnivorous ( $57 \%$ ), eight omnivorous ( $20 \%$ ), five planktivorous ( $13 \%$ ), two herbivorous, and two detritivorous (5\%). Twelve of the carnivorous species were scarce. The only herbivorous species were Nicholsina usta and Sparisoma radiams. The dendrogram showed two main groups: group A, made up of omnivorous and carnivorous species and group B, consisting of detrivorous, herbivorous, and planktivorous species.
\end{abstract}

Key words: fish, Thalassia testudinum, trophic category, Caribbean, Venezuela.

Autor corresponsal: Thays Allen (thayscor@yahoo.com)

* Trabajo presentado en el XXV Congreso de Ciencias del Mar de Chile y XI Congreso Latinoamericano de Ciencias del Mar (COLACMAR), realizados en Viña del Mar, entre el 16 y 20 de mayo de 2005. 


\section{INTRODUCCIÓN}

La fanerógama marina Thalassia testudinum conforma mosaicos de parches vegetados y sustratos libres (Bologna \& Heck, 2000), constituyendo áreas de alta productividad (McRoy \& Macmillan, 1977; Thayer et al., 1984), siendo la fanerógama más abundante y característica del Caribe (Dawes, 1986). Entre sus funciones más sobresalientes está evitar la erosión de las costas, contribuir al enriquecimiento del ambiente donde habita, formando zonas de protección y crianza para invertebrados y peces, estabiliza el fondo marino y representa un área de alimentación para diversos organismos (Randall, 1965; Thayer et al., 1984; Vargas-Maldonado \& Yáñez-Arancibia, 1987).

El valor de las praderas de fanerógamas como fuente de alimento está dado por su disponibilidad, distribución, abundancia, morfología, producción y composición química. Estas praderas tienen un contenido relativamente alto en minerales y otros nutrientes, contenido que varía dependiendo de la parte de la planta, edad, estación y grado de epifitismo (Thayer et al., 1984).

Las praderas de T. testudinum constituyen una fuente importante de alimento a pesar que pocos organismos se alimentan directamente de ella. Sin embargo, las principales cadenas tróficas están basadas en el detrito derivado de esta fanerógama y pueden llegar a sostener cadenas alimentarias de zonas adyacentes; por otro lado, las hojas sirven de substrato a muchos organismos epibiontes que suelen ser consumidos por un gran número de invertebrados y peces. La estructura trófica en una pradera de T. testudinum generalmente está formada por herbívoros, carnívoros, omnívoros, detritívoros y suspensívoros o filtradores, los cuales están interconectados unos a otros por los patrones de flujo energético (Greenway, 1995).

La fauna asociada con T. testudinum es muy variada tanto en vertebrados como invertebrados y desde el punto de vista ecológico y trófico, los diferentes organismos que la habitan son muy importantes. Los peces son los organismos más sobresalientes de esta comunidad debido a que son muy abundantes y utilizan a la fanerógama de diversas maneras, ya sea como refugio o como área de reproducción y alimentación. Las relaciones tróficas son esenciales para inferir sobre las interacciones biológicas en comunidades animales (Carrasson \& Cartes, 2002). La complejidad del hábitat y los depredadores pueden intervenir en la aparición, distribución y abundancia de los pastoreadores (Schanz et al., 2002). La ali- mentación es primordial para la elección del hábitat por los peces y constituye un aspecto importante de su actividad diaria. La particularidad de los peces en los patrones de alimentación viene dado por la locomoción, tipo, distribución, espacio y talla de la fanerógama, y distribución de la presa. Sin embargo, la locomoción muchas veces podría restringir el acceso a varios hábitats y microhábitats modificando el éxito en la captura de la presa (Fulton \& Bellwood, 2002). Para los peces herbívoros su alimentación aparentemente depende de la disrupción de las paredes celulares de los vegetales que consume. Los peces herbívoros que consumen hojas de fanerógamas muchas veces pastorean sus epífitas y solo una pequeña parte de la hoja de Thalassia es utilizada (Thayer et al., 1984).

En el mar Caribe, se han realizado varios estudios sobre la estructura de comunidades de peces en praderas de fanerógamas y sus relaciones tróficas (Randall, 1965, 1967; Greenway, 1976, 1995; Ogden, 1976, 1980; Heck \& Orth, 1980; Lobel \& Ogden, 1981; Guevara-Carrió et al., 1994). En Venezuela, específicamente en el oriente del país, son diversos los estudios sobre los hábitos alimentarios y categorías tróficas de peces (Carreño, 1975; González-Cabello, 1981; Hug, 1984; Parra \& León, 1984; García et al., 1985; Caldera et al., 1988; Parra, 1992; RamírezArredondo, 1994; Rivas et al., 1999). Los estudios sobre hábitos alimentarios y ecología trófica de comunidades de peces asociadas a T. testudinum en Venezuela son muy escasos a pesar de la contribución al conocimiento científico que esto aporta, por lo que se planteó como objetivo principal de este estudio determinar la estructura y categorías tróficas de los peces asociados a tres praderas de T. testudinum del golfo de Cariaco, Venezuela.

\section{MATERIALES Y MÉTODOS}

Se seleccionaron tres praderas de T. testudinum de aguas claras y someras de la costa sur del golfo de Cariaco: Juana Josefa $\left(10^{\circ} 26^{\prime} \mathrm{N}, 6^{\circ} 03^{\prime} \mathrm{W}\right)$, Tocuchare $\left(10^{\circ} 26^{\prime} \mathrm{N}, 64^{\circ} 00^{\prime} \mathrm{W}\right)$ y Tunantal $\left(10^{\circ} 26^{\prime} \mathrm{N}\right.$, $63^{\circ} 59^{\prime} \mathrm{W}$ ) (Fig. 1). El golfo en general presenta una alta productividad debido al fenómeno de surgencia característico del área (Ferraz-Reyes, 1987).

Las capturas de los peces se realizaron de diciembre de 1996 a noviembre de 1997, en horas diurnas por medio de una red tipo chinchorro playero de 1,50 x $50 \mathrm{~m}$ y $0,7 \mathrm{~cm}$ de abertura de malla, realizándose un arrastre mensual en cada pradera. En el labora- 


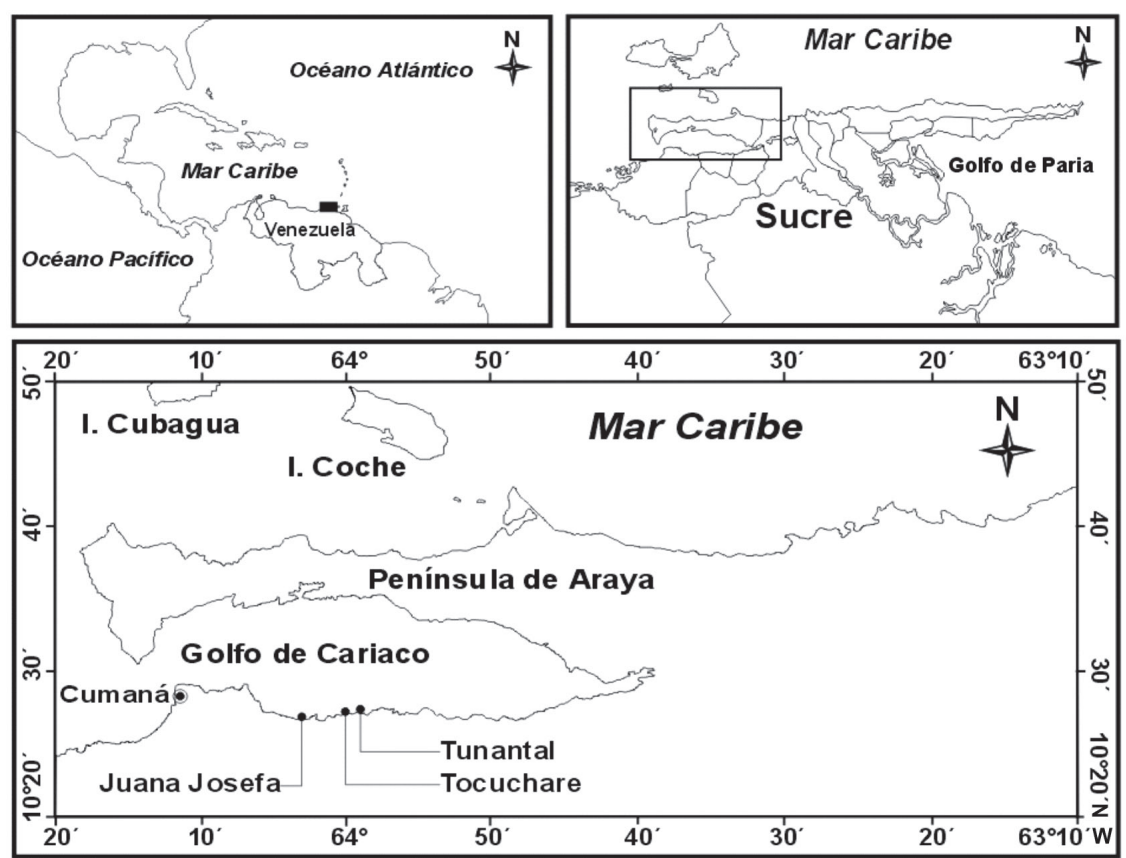

Figura 1. Ubicación de las estaciones de muestreo en el área de estudio.

Figure 1. Sampling stations in the study area.

torio los especímenes se mantuvieron refrigerados a $3^{\circ} \mathrm{C}$. La identificación taxonómica y la ordenación secuencial de las familias se realizaron con las claves de Cervigón (1991, 1993, 1994, 1996) y la página www.fishbase.net. Se contabilizó el número total de especies para obtener la abundancia total de las mismas determinada por el número de individuos capturados por especie $(\mathrm{N})$, la riqueza específica total y frecuencia de ocurrencia, la cual se clasificó en cuatro categorías: persistentes $(\mathrm{P})$ aquellas especies que estuvieron entre 60 y $100 \%$ del total de las capturas; habituales $(\mathrm{H})$ presentes entre 40 y $59 \%$; ocasionales (Oc) presencia entre 20 y $39 \%$, y raras $(\mathrm{R})$ presentes en un porcentaje menor al 19\%. Las especies persistentes utilizan las praderas del golfo durante la mayor parte del año. Las habituales usan las praderas regularmente y podrían depender de ellas para completar algún estadio de su ciclo vital. Las ocasionales y raras son aquellas que aparecen con menor frecuencia en dichas praderas. La categorización descrita es una modificación propuesta por Vargas-Maldonado et al. (1981), para comunidades de peces asociadas a Rhizophora mangle y T. testudinum.

La categoría trófica de cada una de las especies se determinó de acuerdo a los trabajos de varios autores (Randall, 1967; Carreño, 1975; FAO, 1978; González-Cabello, 1981; Lobel \& Orden, 1981;
Vargas-Maldonado et al., 1981; García et al., 1985; Estrada, 1986; Gómez, 1987; Caldera-Rincón et al., 1988; Arenas-Granados \& Acero, 1992; Santos-Martínez \& Arboleda, 1993; Greenway, 1995; SchmitterSoto \& Castro-Aguirre, 1996; Rivas et al., 1999) y se establecieron cinco categorías tróficas: carnívoros, herbívoros, omnívoros, planctívoros y detritívoros de acuerdo a Randall (1967), Vargas-Maldonado et al. (1981) y Livingston (1982).

Para conocer el grado de similitud de las diferentes especies y sus categorías tróficas, se realizó un análisis de conglomerados utilizando el programa estadístico computarizado SPSS 10 y se construyó un dendrograma por afinidad, por el método de centroides, donde cada especie representó un carácter y cada categoría trófica un valor: 1,1 (carnívoro generalizado), 1,2 (carnívoro piscívoro), 1,3 (carnívoro de invertebrados), 2 (omnívoro), 3 (herbívoro), 4 (planctívoro) y 5 (detritívoro).

\section{RESULTADOS}

Se realizaron 36 capturas donde se colectaron 15.509 peces, en su mayoría juveniles pertenecientes a 44 especies y 27 familias. Los resultados de la abundancia, riqueza específica y frecuencia de aparición se 
indican en la sumatoria acumulativa de las 36 capturas. La Tabla 1 indica la lista de familias, especies, abundancias específicas, frecuencia de aparición y el estatus trófico de cada especie. Las especies más abundantes fueron en su mayoría juveniles: Haemulon boschmae (Metzelaar, 1919), Nicholsina usta (Valenciennes, 1839), Atherinella brasiliensis (Quoy \& Gaimard, 1825) (Xenomelaniris brasiliensis, Quoy \& Gaimard, 1824), Diplodus argenteus (Cuvier, 1830), Haemulon steindachneri (Jordan \& Gilbert, 1881), Decapterus macarellus (Cuvier, 1833) y Halichoeres bivittatus (Bloch, 1791) (Tablas 1 y 3). La familia mejor representada, tanto en número de individuos como especies, fue Haemulidae (8.599 ind.; cinco especies), seguida de Carangidae (889 ind.; cuatro especies), Sciaenidae y Paralichthyidae (99 y 54 ind. respectivamente; tres especies cada una). En cuanto a la frecuencia de aparición, $H$. steindachneri, Orthopristis ruber (Cuvier, 1830), $H$. bivittatus y $N$. usta, fueron especies persistentes y representaron el $9,1 \%$ del total de las capturas. $X$. brasiliensis, E. gula (Cuvier, 1830), H. boschmae, D. argenteus, Paralichthys tropicus (Ginsburg, 1933) y Sphoeroides greeleyi (Gilbert, 1900), fueron especies habituales y conformaron el 13,6\% de la captura total. Tilosurus crocodilus (Peron \& Le Sueur, 1821), Syngnatus sp., Bairdiela sanctaeluciae (Jordan, 1899), Labrisomus nuchipinnis (Quoy \& Gaimard, 1824) y Labrisomus sp. fueron ocasionales y les correspondió el $11,4 \%$ del total y las 29 especies restantes fueron especies raras formando el $65,9 \%$ de la recolección total (Tabla 1).

Se reporta la clasificación de 40 especies de peces indicando su categoría trófica según diversos autores, faltando determinar los hábitos alimentarios de Syngnathus sp., Citharichthys sp., P. tropicus y Etropus crossotus (Jordan \& Gilbert, 1882) por falta de información (Tablas 2 y 3). Se determinaron 23 especies carnívoras $(57 \%)$, ocho omnívoras $(20 \%)$, cinco planctívoras $(13 \%)$, dos herbívoras y dos detritívoras ( $5 \%$ cada una) (Tabla 1). Las especies carnívoras (23) fueron las mejor representadas en cuanto a especies, lo que permitió agruparlas en tres categorías: carnívoros generalizados (consumen invertebrados y pequeños peces), carnívoros piscívoros (consumen sólo peces) y carnívoros de invertebrados (consumen sólo invertebrados, como moluscos, crustáceos, poliquetos, etc). Del total, ocho resultaron carnívoras generalizadas (35\%), 10 carnívoras de invertebrados (43\%) y cinco carnívoras piscívoras (22\%). Sólo cuatro especies se presentaron con una frecuencia de aparición persistente $N$. usta (herbívora); O. ruber y H. bivittatus (carnívoras) y H. steindachneri (planctívora). La Tabla 3 indica las especies juveniles capturadas más abundantes con sus categorías tróficas, la longitud máxima $(\mathrm{cm}$ LT) (datos obtenidos de www.fishbase.net) y rango de talla. De las 23 especies carnívoras capturadas en este trabajo 12 fueron ocasionales. Las únicas especies herbívoras fueron $N$. usta y Sparisoma radiams (Valenciennes, 1840) (Tabla 1).

El análisis de conglomerado, a partir de los datos de las categorías tróficas de las 40 especies de peces, permitió reconocer dos grupos principales. El grupo A, constituido por dos subgrupos: representados por especies omnívoras (a) y especies carnívoras (b). El grupo B, conformado por tres subgrupos, que incluye las especies detritívoras (c), herbívoras (d) y planctívoras (e) (Fig. 2).

\section{DISCUSIÓN}

Las comunidades de praderas de Thalassia testudinum son áreas utilizadas por muchas especies, caracterizadas por la alta abundancia y diversidad de peces. El considerable número de especies de peces juveniles que se registra en esta investigación (44), indicaría que ellos utilizan la zona de diferentes formas. De las 40 especies de peces estudiadas tróficamente, 57\% son carnívoras y $20 \%$ omnívoras. El alto porcentaje de carnívoros en la zona, seguido de los omnívoros, se debe posiblemente a que las praderas representan un verdadero acopio de numerosos invertebrados (tales como moluscos, crustáceos, poliquetos, etc.) (Randall, 1965; Jiménez, 1994; Greenway, 1995) y peces que pudieran servir de alimento a esos organismos. Estos resultados sugieren la eficiente utilización por parte de la ictiofauna de la oferta energética que conceden las praderas de T. testudinum.

Con respecto a las especies más abundantes, se observa que los hábitos alimentarios de los haemúlidos varían de juveniles a adultos; la dieta de los primeros está constituida por pequeños organismos planctónicos, como copépodos, larvas de crustáceos y en general, los juveniles se alimentan de día a excepción de $H$. chrysargyreum que lo realiza de noche (Estrada, 1986). Orthopristis ruber, es considerado un pez carnívoro y depredador importante de crustáceos, pero no se descarta que se alimente de moluscos, poliquetos, pequeños peces, etc. (González-Cabello, 1981). Los gérridos son peces de importancia económica y ecológica que al igual que otros organismos, cumplen una función definida en 


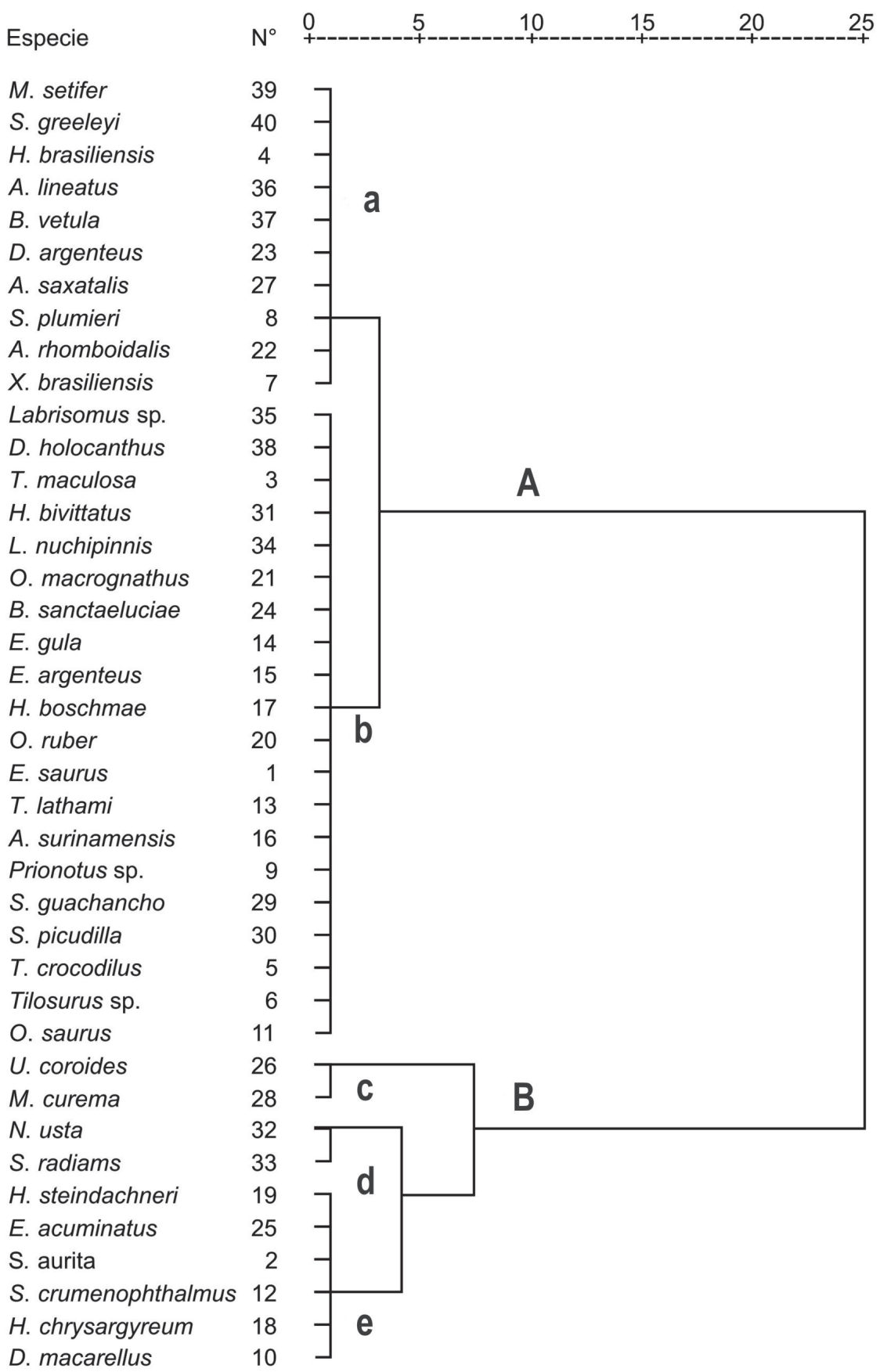

Figura 2. Dendrograma de similitud que agrupa las distintas especies de peces asociados con Thalassia testudinum basado en sus categorías tróficas (carnívoros, omnívoros, herbívoros, planctívoros y detritívoros).

Figure 2. Similarity dendrogram of different fish species associated with Thalassia testudinum based on its trophics categories (carnivorous, omnivorous, herbivorous, planktivorous and detritivorous). 
Tabla 1. Familias, especies, abundancia específica $(\mathrm{N})$, frecuencia de aparición (P: persistentes, con presencia entre 60-100\% del total de las caladas; $H$ : habitual, entre 59-40\%; Oc: ocasional; entre 39-20\% y R: raras presentes en porcentaje menor al 19\%), estatus trófico (C: carnívoro, P: planctívoro, O: omnívoro, D: detritívoro, H: herbívoro); diversidad total (bits/ind) y equitabilidad total.

Table 1. Families, species, specific abundances (n), appearance frequency (P: persistent, with presence from 60 to $100 \%$ of the total, H: habitual, from 40 to 59\%; Oc: occasional; from 20 to $39 \%$ and R: rare, presents in smaller percentage less to $19 \%$ ), trophics status (C: carnivorous, P: planktivorous, O: omnivorous, D: detritivorous, H: herbivorous); total diversity (bits/ind) and total evenness.

\begin{tabular}{|c|c|c|c|c|}
\hline Familia & Especie & $\begin{array}{l}\text { Número de } \\
\text { individuos }\end{array}$ & $\begin{array}{c}\text { Frecuencia } \\
\text { aparición }\end{array}$ & $\begin{array}{c}\text { Hábitos } \\
\text { alimentarios }\end{array}$ \\
\hline Elopidae & Elops saurus & 2 & $\mathrm{R}$ & $\mathrm{C}$ \\
\hline Clupeidae & Sardinella aurita & 30 & $\mathrm{R}$ & $\mathrm{P}$ \\
\hline Batrachoididae & Thalassophryne maculosa & 3 & $\mathrm{R}$ & $\mathrm{C}$ \\
\hline Hemirhamphidae & Hemirhamphus brasiliensis & 7 & $\mathrm{R}$ & $\mathrm{O}$ \\
\hline \multirow[t]{2}{*}{ Belonidae } & Tilosurus crocodilus & 11 & Oc & $\mathrm{C}$ \\
\hline & Tilosurus sp. & 1 & $\mathrm{R}$ & $\mathrm{C}$ \\
\hline Atherinopsidae & Atherinella brasiliensis & 1190 & $\mathrm{H}$ & $\mathrm{O}$ \\
\hline Syngnathidae & Syngnathus sp. & 8 & Oc & -- \\
\hline Scorpaenidae & Scorpaena plumieri & 1 & $\mathrm{R}$ & $\mathrm{O}$ \\
\hline Triglidae & Prionotus sp. & 96 & $\mathrm{R}$ & $\mathrm{C}$ \\
\hline \multirow[t]{4}{*}{ Carangidae } & Decapterus macarellus & 879 & $\mathrm{R}$ & $\mathrm{P}$ \\
\hline & Oligoplites saurus & 4 & $\mathrm{R}$ & $\mathrm{C}$ \\
\hline & Selar crumenophthalmus & 1 & $\mathrm{R}$ & $\mathrm{P}$ \\
\hline & Trachurus lathami & 5 & $\mathrm{R}$ & $\mathrm{C}$ \\
\hline \multirow[t]{2}{*}{ Gerreidae } & Eucinostomus gula & 542 & $\mathrm{H}$ & $\mathrm{C}$ \\
\hline & Eucinostomu argenteus & 14 & $\mathrm{R}$ & $\mathrm{C}$ \\
\hline \multirow[t]{5}{*}{ Haemulidae } & Anisotremus surinamensis & 9 & $\mathrm{R}$ & $\mathrm{C}$ \\
\hline & Haemulon boschmae & 5700 & $\mathrm{H}$ & $\mathrm{C}$ \\
\hline & Haemulon chrysargyreum & 138 & $\mathrm{R}$ & $\mathrm{P}$ \\
\hline & Haemulon steindachneri & 903 & $\mathrm{P}$ & $\mathrm{P}$ \\
\hline & Orthopristis ruber & 1849 & $\mathrm{P}$ & $\mathrm{C}$ \\
\hline Opistognathidae & Opistognathus macrognathus & 1 & $\mathrm{R}$ & $\mathrm{C}$ \\
\hline \multirow{2}{*}{ Sparidae } & Archosargus rhomboidalis & 1 & $\mathrm{R}$ & $\mathrm{O}$ \\
\hline & Diplodus argenteus & 1161 & $\mathrm{H}$ & $\mathrm{O}$ \\
\hline \multirow{3}{*}{ Scianidae } & Bairdiella sanctaeluciae & 92 & Oc & $\mathrm{C}$ \\
\hline & Equetus acuminatus & 1 & $\mathrm{R}$ & $\mathrm{C}$ \\
\hline & Umbrina coroides & 6 & $\mathrm{R}$ & $\mathrm{D}$ \\
\hline Pomacentridae & Abudefduf saxatalis & 4 & $\mathrm{R}$ & $\mathrm{O}$ \\
\hline Mugilidae & Mugil curema & 6 & $\mathrm{R}$ & $\mathrm{D}$ \\
\hline \multirow[t]{2}{*}{ Sphyraenidae } & Sphyraena guachancho & 1 & $\mathrm{R}$ & $\mathrm{C}$ \\
\hline & Sphyraena picudilla & 19 & $\mathrm{R}$ & $\mathrm{C}$ \\
\hline Labridae & Halichoeres bivittatus & 800 & $\mathrm{P}$ & $\mathrm{C}$ \\
\hline \multirow[t]{2}{*}{ Scaridae } & Nicholsina usta & 1876 & $\mathrm{P}$ & $\mathrm{H}$ \\
\hline & Sparisoma radiams & 1 & $\mathrm{R}$ & $\mathrm{H}$ \\
\hline \multirow[t]{2}{*}{ Labrisomidae } & Labrisomus nuchipinnis & 33 & Oc & $\mathrm{C}$ \\
\hline & Labrisomus sp. & 21 & $\mathrm{Oc}$ & $\mathrm{C}$ \\
\hline Achiridae & Achirus lineatus & 2 & $\mathrm{R}$ & $\mathrm{O}$ \\
\hline \multirow{3}{*}{ Paralichthydae } & Citharichthys sp. & 2 & $\mathrm{R}$ & -- \\
\hline & Paralichthys tropicus & 51 & $\mathrm{H}$ & -- \\
\hline & Etropus crossotus & 1 & $\mathrm{R}$ & -- \\
\hline Balistidae & Balistes vetula & 3 & $\mathrm{R}$ & $\mathrm{O}$ \\
\hline Diodontidae & Diodon holocanthus & 1 & $\mathrm{R}$ & $\mathrm{C}$ \\
\hline Monacantidae & Monacanthus setifer & 3 & $\mathrm{R}$ & $\mathrm{O}$ \\
\hline Tetraodontidae & Sphoeroides greeleyi & 30 & $\mathrm{H}$ & $\mathrm{C}$ \\
\hline Total de individuos & & 15.509 & & \\
\hline Total de especies & & 44 & & \\
\hline Total de familias & & 27 & & \\
\hline
\end{tabular}


la red trófica de los ecosistemas marinos. El espectro trófico de E. gula es amplio, siendo principalmente un consumidor de pequeños invertebrados bénticos, donde los crustáceos son preferidos por la especie (Arenas-Granado \& Acero, 1992; Rivas et al., 1999). Atherinella brasiliensis, en las costas de Venezuela presenta una alimentación variada y cambiante de juvenil a adulto (poliquetos, quetognatos, crustáceos, anfípodos, insectos, moluscos, gastrópodos, restos de algas, etc.), lo que indica que es una especie omnívora, pero su dieta está compuesta principalmente por crustáceos, copépodos y cirrípedos (Carreño, 1975). $N$. usta, especie muy abundante en las praderas de T. testudinum del oriente del país (Gómez, 1987), es considerada una especie herbívora que ingiere principalmente Thalassia como otros escáridos (Randall, 1965, 1967; Weinstein \& Heck, 1979).

Se puede señalar que los invertebrados y epibiontes de las hojas de T. testudinum desempeñan un papel importante en la dieta de los peces omnívoros y carnívoros, debido al flujo de energía que esto implica en la estructura trófica de una comunidad dada. En este sentido, Greenway (1995) reportó que los crustáceos fueron la principal fuente de alimento tanto para omnívoros como carnívoros y representa un importante rubro en el flujo de energía, particularmente en los niveles intermedios de la estructura trófica. El consumo directo de la fanerógama aporta muchos nutrientes para la dieta de los peces omnívoros debido a los altos niveles de carbohidratos solubles que incrementan la eficiencia metabólica y además, podría ser energéticamente favorable para algunos carnívoros (Montgomery \& Targett, 1992).

Los peces carnívoros constituyen generalmente los más importantes y diversos organismos que habitan las comunidades demersales (Randall, 1965, 1967). La caracterización trófica de un conjunto de peces en una localidad dada está determinada por la disponibilidad de la presa y la complejidad estructural del hábitat donde se encuentren, el cual no solo pudiera dar refugio sino también ser fuente directa e indirecta de alimento (Angel \& Ojeda, 2001).

Se pudiera pensar que la competencia interespecífica por alimento es alta debido al alto porcentaje de peces que presentaron la misma categorización trófica, además en su mayoría fueron especies raras, lo que indicaría que las praderas en estudio se comportan más que como un refugio, como un área de alimentación donde los peces llegan a ellas en diferentes momentos por la preferencia de alimento y disponibilidad de la presa. MacPherson (1981) evidenció para varias asociaciones de peces que el alimento es un importante mecanismo de segregación ecológica en un momento determinado. La competencia interespecífica se minimiza cuando la densidad, abundancia y distribución de la presa es elevada, favoreciendo la coexistencia de especies de peces en un área determinada (Arenas-Granado \& Acero, 1992). Al respecto, Valtierra-Vega \& Schmitter-Soto (2000), señalan para siete especies de peces cíclidos que la aparente falta de competencia por alimento se atribuiría a que los recursos alimentarios son abundantes en el ecosistema. Allen \& Jiménez (2001), observaron que la mayoría de los peces llegaban eventualmente a las praderas de Thalassia testudinum utilizando dichas áreas en horas diurnas para su alimentación y protección.

El bajo porcentaje de especies herbívoras, indicaría que a pesar que las praderas de T. testudinum son productores primarios, su consumo directo está limitado a algunos herbívoros, ya que la mayoría de los organismos se alimenta de los epifitos de la planta. Posiblemente esto se deba a que en el área no abundan otros ítemes vegetales, como algas, otra fanerógama, etc., que sean consumidos por otros peces herbívoros, limitando así la llegada de otras especies a la zona. Por otra parte, es posible que existieran otras especies herbívoras de hábitos alimentarios nocturnos, que no pudieron ser capturadas durante el día, por lo tanto, no se puede asegurar que en las praderas estudiadas existan pocas especies herbívoras. La preferencia por T. testudinum como alimento principal en su dieta posiblemente fue lo que hizo que $N$. usta y $S$. radiams aparecieran como los únicos peces herbívoros en el área.

Se consideran a estos escáridos herbívoros, habitantes de las praderas de Thalassia que tienen actividad diurna y nocturna (Valdés-Muñoz, 1987; Méndez et al., 1988). Nicholsina usta es una especie característica de praderas de $T$. testudinum de áreas de surgencia que ingiere dicha planta como otros escáridos y $S$. radiams cumple la misma función que N. usta en otras áreas del Caribe (Gómez, 1987). Consideraciones similares fueron hechas por Méndez et al. (1988), quienes mencionan a $N$. usta como el escárido de más amplia distribución en la bahía de Mochima indicando que es una especie que cumple todo su ciclo vital en las praderas de Thalassia. Lobel \& Ogden (1981) mencionan a $S$. radiams como una especie exclusivamente herbívora, que se alimenta de las hojas de Thalassia y come las partes viejas de la planta cubiertas con epifitos, señalan además que 
Tabla 2. Clasificación de las especies de peces indicando su categoría trófica según diversos autores.

Table 2. Species classification of fishes indicating its trophics category according to several authors.

\begin{tabular}{|c|c|c|}
\hline Especies & $\begin{array}{l}\text { Categoría } \\
\text { trófica }\end{array}$ & Autor \\
\hline Elops saurus & Carnívoro & Santos-Martínez \& Arboleda, 1993 \\
\hline Thalassophryne maculosa & & FAO, 1978 \\
\hline Tilosurus crocodilus & & Randall, 1967; FAO, 1978 \\
\hline Tilosurus sp. & & Randall, 1967; FAO, 1978 \\
\hline Scorpaena plumieri & & Randall, 1967; FAO, 1978; Greenway, 1995 \\
\hline Prionotus sp. & & FAO, 1978; Schmitter-Soto \& Castro-Aguirre, 1996 \\
\hline Oligoplites saurus & & Randall, 1967; Vargas-Maldonado et al., 1981 \\
\hline Trachurus lathami & & FAO, 1978 \\
\hline Eucinostomus gula & & $\begin{array}{l}\text { FAO, 1978; Greenway, 1995; Arenas-Granado \& Acero, 1992; } \\
\text { Rivas et al., } 1999\end{array}$ \\
\hline Eucinostomи argenteus & & $\begin{array}{l}\text { Randall, 1967; FAO, 1978; Arenas-Granado \& Acero, 1992; } \\
\text { Rivas et al., } 1999\end{array}$ \\
\hline Anisotremus surinamensis & & Randall, 1967; FAO, 1978 \\
\hline Haemulon boschmae & & FAO, 1978 \\
\hline Orthopristis ruber & & FAO, 1978; González-Cabello, 1981 \\
\hline Opistognathus macrognathus & & Randall, 1967 \\
\hline Bairdiella sanctaeluciae & & FAO, 1978 \\
\hline Equetus acuminatus & & Randall, 1967; Vargas-Maldonado et al., 1981 \\
\hline Sphyraena guachancho & & Randall, 1967; FAO, 1978 \\
\hline Sphyraena picudilla & & Randall, 1967; FAO, 1978 \\
\hline Halichoeres bivittatus & & Randall, 1967 \\
\hline Labrisomus nuchipinnis & & Randall, 1967 \\
\hline Labrisomus sp. & & Randall, 1967 \\
\hline Diodon holocanthus & & Randall, 1967; Greenway, 1995 \\
\hline Sphoeroides greeleyi & & FAO, 1978; Vargas-Maldonado et al., 1981 \\
\hline Hemirhamphus brasiliensis & Omnívoro & Randall, 1967; FAO, 1978 \\
\hline Atherinella brasiliensis & & Carreño, 1975 \\
\hline Archosargus rhomboidalis & & Randall, 1967; FAO, 1978; Greenway, 1995 \\
\hline Diplodus argenteus & & Randall, 1967; FAO, 1978 \\
\hline Abudefduf saxatalis & & Randall, 1967; FAO, 1978 \\
\hline Achirus lineatus & & Vargas-Maldonado et al., 1981; Greenway, 1995 \\
\hline Balistes vetula & & Randall, 1967 \\
\hline Monacanthus setifer & & Randall, 1967 \\
\hline Sardinella aurita & Planctívoro & FAO, 1978; García et al., 1985; Caldera- Rincón et al., 1988 \\
\hline Decapterus macarellus & & Randall, 1967; FAO, 1978 \\
\hline Selar crumenophthalmus & & Randall, 1967; FAO, 1978 \\
\hline Haemulon chrysargyreum & & Randall, 1967; Estrada, 1986 \\
\hline Haemulon steindachneri & & FAO, 1978; Estrada, 1986 \\
\hline Umbrina coroides & Detritívoro & FAO, 1978 \\
\hline Mugil curema & & Randall, 1967; FAO, 1978 \\
\hline Nicholsina usta & Herbívoro & Randall, 1967; FAO, 1978; Gómez, 1978 \\
\hline Sparisoma radiams & & Randall, 1967; Greenway, 1995; Lobel \& Orden, 1981 \\
\hline
\end{tabular}


Tabla 3. Especies de peces más abundantes con sus categorías tróficas, número de individuos, longitud máxima (LT en cm) y rango de tallas de sus capturas.

Table 3. More abundant fish species with its trophics categories, number of individuals, maximal length (cm LT) and the size range of capture.

\begin{tabular}{llccc}
\hline Especies & Categorías & $\begin{array}{c}\text { Número de } \\
\text { individuos }\end{array}$ & $\begin{array}{c}\text { Longitud máxima } \\
\text { (LT en cm) }\end{array}$ & $\begin{array}{c}\text { Rango de talla } \\
\text { (LT en cm) }\end{array}$ \\
\hline Eucinostomus gula & Carnívoros & 542 & 23 & $6,0-14,0$ \\
Orthopristis ruber & & 1849 & 40 & $3,5-13,0$ \\
Haemulon boschmae & & 5700 & 19 & $3,0-8,0$ \\
Halichoeres bivittatus & & 800 & 35 & $5,4-18,2$ \\
\hline Atherinella brasiliensis & Omnívoros & 1190 & 16 & $4,5-7,0$ \\
Diplodus argenteus & & 1161 & 29,2 & $4,0-10,2$ \\
\hline Haemulon steindachneri & Plantívoros & 903 & 30 & $2,5-10,8$ \\
Decapterus macarellus & & 879 & 46 & $5,4-10,6$ \\
\hline Nicholsina usta & Herbívoro & 1876 & 30 & $6,0-14,8$ \\
\hline
\end{tabular}

la selección de la planta pareciera ser independiente de la abundancia relativa de la misma. Este mismo escárido fue la única especie herbívora consumidora de hojas de Thalassia reconocida por Greenway (1995) en el Caribe; en este mismo sentido Kirsch et al. (2002), señalan a los escáridos como consumidores voraces de T. testudinum.

Es importante destacar que el dendrograma se construyó en función de los hábitos alimentarios de las diferentes especies de peces. No se emplearon criterios cuantitativos, los que en su momento pudieran definir más ampliamente la relación de las diferentes especies y lo que ellos comen específicamente. Sin embargo, el análisis refleja la existencia para las costas orientales de un número considerable de especies de peces que pudieran estar alimentándose solo de los recursos que le proporcionan las praderas de $T$. testudinum. Las interpretaciones de tales resultados son parciales, pero permiten aproximarse a los principios generales que regulan el comportamiento de la comunidad en tiempo y espacio. El análisis señala una clara separación de las muestras, que parece estar asociada con la composición de la ictiofauna, su interacción con el hábitat y a la disponibilidad de alimento. Posiblemente la combinación de aguas tranquilas, efecto de surgencia, abundancia y disponibilidad de recursos alimentarios, hace que esta área sea un buen criadero y centro de alimentación. Por lo que se presume que los peces encuentran en estos pastos no sólo protección, sino un verdadero arsenal de alimento. La combinación de diferentes factores bióticos y abióticos hace que los hábitats de pastos marinos y manglar ofrezcan numerosas ventajas en el uso de estos ecosistemas (Yáñez-Arancibia et al., 1991, 1993). El alto número de peces en praderas podría ser causado por una variedad de procesos, incluyendo la complejidad del hábitat, suministro de alimento, refugio para la depredación y efectos hidrodinámicos (Hindell et al., 2001). Al respecto, Angel \& Ojeda (2001), mencionan que la complejidad del hábitat influye en la estructura y organización trófica de la comunidad.

\section{AGRADECIMIENTOS}

Al Instituto Oceanográfico de Venezuela en la Universidad de Oriente, Núcleo de Sucre. Al personal técnico de laboratorio y captura, Carlos Figueroa y Fidel Marchan, al Sr. Alejandro de La Rosa, técnico marino. A los profesores Nieves Aguado y Oscar Díaz por la lectura crítica y recomendaciones al manuscrito.

\section{REFERENCIAS}

Allen, T. \& M. Jiménez. 2001. Comunidad de peces en tres praderas de Thalassia testudinum del golfo de Cariaco, Estado de Sucre, Venezuela. Bol. Inst. Oceanogr. Venezuela, Univ. Oriente, 40(1-2): 3948.

Angel, A. \& P.F. Ojeda. 2001. Structure and trophic organization of subtidal fish assemblages on the northern Chilean coast: the effect of habitat complexity. Mar. Ecol. Progr. Ser., 217: 81-91. 
Arenas-Granados, P. \& A. Acero. 1992. Organización trófica de las mojarras (Pisces: Gerreidae) de la Ciénaga Grande de Santa Marta (Caribe colombiano). Rev. Biol. Trop., 40(3): 287-302.

Bologna, P.A. \& K.L. Heck, Jr. 2000. Impacts of seagrass habitat architecture on bivalve settlement. Estuaries, 23(4): 449-457.

Caldera-Rincón, M., M.F. Hug \& I. Ramírez-Arredondo. 1988. Aspectos alimenticios de la sardina, Sardinella aurita Valenciennes, 1847 (Pisces: Clupeidae) de los alrededores de la región noroccidental de la península de Araya y alrededores de las islas de Coche y Cubagua, Venezuela. Bol. Inst. Oceanogr. Venezuela, Univ. Oriente, 27(1-2): 129-143.

Carreño, R. 1975. Algunos aspectos de la biología del tinícalo, Xenomelaniris brasiliensis (Quoy \& Gaimard, 1824) del golfo de Cariaco, Estado de Sucre (Pisces: Atherinidae). Tesis de Biología. Universidad de Oriente, Venezuela, 43 pp.

Carrasson, M. \& J.E. Cartes. 2002. Trophic relationships in a mediterranean deep-sea fish community partition of food resources, dietary overlap and connections within the benthic boundary layer. Mar. Ecol. Progr. Ser., 241: 41-55.

Cervigón, F. 1991. Los peces marinos de Venezuela. Fundación Científica de los Roques. Vol. I, 425 pp.

Cervigón, F. 1993. Los peces marinos de Venezuela. Fundación Científica de los Roques. Vol. II, 498 pp.

Cervigón, F. 1994. Los peces marinos de Venezuela. Fundación Científica de los Roques. Vol. III, 295 pp.

Cervigón, F. 1996. Los peces marinos de Venezuela. Fundación Científica de los Roques. Vol. IV, 255 pp.

Dawes, C. 1986. Botánica marina. Editorial Limusa, México, 673 pp.

Estrada, M. 1986. Hábitos alimentarios de los peces del género Haemulon (Pisces: Haemulidae) de los arrecifes de la región de Santa Marta, Colombia. An. Invest. Mar. Punta Betín, 15-16: 49-66.

Organización de Naciones Unidas para la Agricultura y la Alimentación (FAO). 1978. Species identification sheets for fishery purposes. Western Central Atlantic (Fishing Area 31). Editado por: W. Fischer. Marine Resources Service. Fishery Resources and Environment Division. FAO Fishery Department.
Volumen II, III, IV y V. Food and Agriculture Organization of the United Nations, Rome.

Ferraz-Reyes, E. 1987. Productividad primaria del golfo de Cariaco, Venezuela. Bol. Inst. Oceanogr. Venezuela, Univ. Oriente, 26(1-2): 97-110.

Filgueira López, E. 2001. Análisis de datos con SPSS Win (SPSS versión 10,0 en español). Editorial Alianza, Madrid, 200 pp.

Froese, R. \& D. Pauly (eds.). 2006. FishBase. World Wide Web electronic publication. www.fishbase. org., Version 06/2006.

Fulton, Ch.J. \& D.R. Bellwood. 2002. Patterns of foranging in labrid fishes. Mar. Ecol. Progr. Ser., 226: $135-142$.

García, O., M.F. Hug \& I. Ramírez-Arredondo. 1985. Aspectos alimenticios de la sardina Sardinella aurita Valenciennes, 1847 (Pisces: Clupeidae) de los alrededores de la isla de Margarita, Estado Nueva Esparta, Venezuela. Bol. Inst. Oceanogr. Venezuela, Univ. Oriente, 24(1-2): 31-42.

Gómez, A. 1987. Estructura de la taxocenosis de peces en praderas de Thalassia testudinum de la bahía de Charagato, isla de Cubagua, Venezuela. Bol. Inst. Oceanogr. Venezuela, Univ. Oriente, 26 (1-2): 125-146.

González-Cabello, L.W. 1981. Régimen alimentario del corocoro, Orthopristis ruber (Cuvier, 1830) (Pisces: Pomadasyidae) en las zonas adyacentes a la isla de Margarita, Venezuela. Bol. Inst. Oceanogr. Venezuela, Univ. Oriente, 20(1-2): 23-32.

Guevara-Carrió, E., A. Bosch-Méndez, R. SuárezMulkay \& R. Lalana-Rueda. 1994. Alimentación natural de tres especies de pargos (Pisces: Lutjanidae) en el archipiélago de los Canarreos, Cuba. Rev. Invest. Mar., 15(4): 63-72.

Greenway, M. 1976. The grazing of Thalassia testudinum in Kingston harbour, Jamaica. Aquat. Bot., 2: $117-126$.

Greenway, M. 1995. Trophic relationships of macrofauna within a Jamaican seagrass meadow and the role of the echinoid Lytechinus variegates (Lamarck). Bull. Mar. Sci., 56: 719-736.

Heck, K.L. Jr. \& R.J. Orth. 1980. Seagrass habitats: the roles of habitat complexity, competition, and predation in structuring associated fish and motile macroinvertebrate assemblages. En: V.S. Kennedy (ed.). Estuarine perspectives. Academic Press, New York, pp. 449-464. 
Hindell, J.S., G.P. Jenkins \& M.J. Keough. 2001. Spatial and temporal variability in the effects of fish predation on macrofauna in relation to habitat complexity and cage effects. Mar. Ecol. Progr. Ser., 224: 231-250.

Hug, M. 1984. Alimentación de las dos especies de anchovetas Anchoa lyolepsis (Evermamm \& Marsh) y A. lamprotaenia (Hildebrand) (Pisces: Engraulidae) del golfo de Cariaco, Venezuela. Bol. Inst. Oceanogr. Venezuela, Univ. Oriente, 23(1-2): 149-156.

Jiménez, M. 1994. Comunidad de moluscos asociadas a Thalassia testudinum, Ensenada de Reyes, bahía de Mochima, Estado de Sucre, Venezuela. Inst. Oceanogr. Venezuela, Univ. Oriente, 33(1-2): 67-76.

Kirsch, K.D., J.F. Valentine \& K.L. Heck Jr. 2002. Parrotfish grazing on turtlegrass Thalassia testudinum: evidence for the importance of seagrass consumption in food web dynamics of the Florida Keys National Marine Sanctury. Mar. Ecol. Progr. Ser., 227: 71-85.

Livingston, R. 1982. Trophic organization of fishes in a coastal seagrass system. Mar. Ecol. Progr. Ser., 7: $1-12$.

Lobel, P.S. \& J.C. Ogden. 1981. Foraging by the herbivorous parrotfish Sparisoma radiams. Mar. Biol., 64: 73-183.

MacPherson, E. 1981. Resource partitioning in a Mediterraneam demersal fish community. Mar. Ecol. Progr. Ser., 4: 183-193.

McRoy, C.P. \& C. McMillan. 1977. Productivity and physiological ecology of seagrasses. En: C.P. McRoy \& C. Helfferich (eds.). Seagrass ecosystems: a scientific perspective. Marcel Dekker, New York, pp. 53-88.

Méndez, E., R. Manrique \& F. Cervigón. 1988. La ictiofauna de la bahía de Mochima. Estación de Investigaciones Marinas de Mochima, FUNDACIENCIAS, $127 \mathrm{pp}$.

Montgomery, J.L.M. \& T.E. Targett. 1992. The nutritional role of seagrass in the diet of the omnivoros pintsh Lagodon rhomboides (L). J. Exp. Mar. Biol. Ecol., 158: 37-57.

Ogden, J.C. 1976. Some aspects of herbivore-plant relations in Caribbean reefs and seagrass beds. Aquat. Bot., 2: 103-116.

Ogden, J.C. 1980. Faunal relationships in Caribbean seagrass beds. En: R.C. Phillips \& C.P. McRoy (eds.). Handbook of seagrass biology, an ecosystem perspective. Garland STPM Press, New York, pp. 173-198.

Parra, B. 1992. Análisis del contenido estomacal y hábitos alimenticios de Paralabrax dewegeri (Pisces: Serranidae) de los alrededores de la isla de Cubagua y punta de Araya, Venezuela. Bol. Inst. Oceanogr. Venezuela, Univ. Oriente, 31(1-2): 73-89.

Parra, B. \& J. León. 1984. Análisis del contenido estomacal y hábitos alimenticios de la Camiguana Anchoa parva del golfo de Cariaco, Estado de Sucre, Venezuela. Bol. Inst. Oceanogr. Venezuela, Univ. Oriente, 23(1-2): 3-13.

Ramírez-Arredondo, I. 1994. Aspectos alimenticios de la cachama pintada, Euthynnus alletteratus (Pisces: Scombidae) de los alrededores de la isla Picua, Estado de Sucre, Venezuela. Bol. Inst. Oceanogr. Venezuela, Univ. Oriente, 33(1-2): 113-120.

Randall, J.E. 1965. Grazing effect on sea grasses by herbivorous reef fishes in the West Indies. Ecology, 46: 255-260.

Randall, J.E. 1967. Food habits of reef fishes of the west indies. Stud. Trop. Oceanogr., 5: 665-847.

Rivas, A., E. Méndez, A. Torres \& L. Martínez. 1999. Hábitos alimenticios de Eucinostomus gula y Eucinostomus argenteus (Pisces: Gerreidae), bahía de Mochima, Estado de Sucre, Venezuela. Bol. Inst. Oceanogr. Venezuela, Univ. Oriente, 38(2): 91-97.

Santos-Martínez, A. \& S. Arboleda. 1993. Aspectos biológicos y ecológicos del Macabí Elops saurus Linnaeus (Pisces: Elopidae) en la Ciénaga Grande de Santa Marta y costa adyacente, Caribe colombiano. An. Inst. Invest. Mar. Punta Betín, 22: 77-96.

Schmitter-Soto, J.J. \& J.L. Castro-Aguirre. 1996. Trophic comparison among Triglidae (Pisces: Scorpaeniformes) off Baja California Sur, México. Rev. Biol. Trop., 44(2): 803-811.

Schanz, A., P. Polte \& H. Asmus. 2002. Cascading effects of hydrodinamics on an epiphyte-grazer system in intertidal seagrass beds of the Wadden Sea. Mar. Biol., 141: 287-297.

Thayer, G.W., K.A. Bjorndal, J.C. Ogden, S.L. Williams, J.C. Ogden \& J.C. Zieman. 1984. Role of larger herbivores in seagrass communities. Estuaries, 7(4): 351-376.

Valdés-Muñoz, E. 1987. Conducta diurna-nocturna de la ictiofauna de los manglares y zonas adyacentes. Acad. Cien. Cuba, Reporte de Investigación del Instituto de Oceanología, 60: 1-16. 
Valtierra-Vega, M.T. \& J.J. Schmitter-Soto. 2000. Hábitos alimentarios de las mojarras (Perciformes: Cichlidae) de la laguna Caobas, Quintana Roo, México. Rev. Biol. Trop., 48(2/3): 503-508.

Vargas-Maldonado, I. \& A. Yáñez-Arancibia. 1987. Estructura de las comunidades de peces en sistemas de pastos marinos (Thalassia testudinum) de la laguna de Término, Campeche, México. An. Inst. Cienc. del Mar y Limnol., Univ. Nal. Autón. México, 14(2): 181-196.

Vargas-Maldonado, I., A. Yáñez-Arancibia \& F. Amezcua-Linares. 1981. Ecología y estructura de las comunidades de peces en áreas de Rhizophora mangle y Thalassia testudinum de la isla del Carmen, laguna de Términos sur del golfo de México. An. Inst. Cienc. del Mar y Limnol., Univ. Nal. Autón. México, 8(1): 241-266.

Recibido: 28 abril 2006; Aceptado: 23 octubre 2006
Weinstein, M.P. \& K.L. Heck Jr. 1979. Ichthyofauna of seagrass meadows along the Caribbean coast of Panamá and in the gulf of Mexico: Composition, structure and community ecology. Mar. Biol., 50: 97-107.

Yáñez-Arancibia, A., A.L. Lara-Domínguez \& J.W. Day. 1993. Interactions between mangrove and seagrass habitats mediated by estuarine nekton assemblages: coupling of primary and secondary production. Hidrobiología, 264: 1-12.

Yáñez-Arancibia, A., P. Sánchez-Gil \& A.L. LaraDomínguez. 1991. Interacciones ecológicas estuario-mar: estructura funcional de bocas estuarinas y su efecto en la productividad del ecosistema. Academia de Ciencias São Paulo. Publ. ACIESP, 73: 1-35. 